Vietnam Journal of Mechanics, VAST, Vol. 42, No. 1 (2020), pp. 43 -61

DOI: https://doi.org/10.15625/0866-7136/14628

\title{
LARGE DISPLACEMENTS OF FGSW BEAMS IN THERMAL ENVIRONMENT USING A FINITE ELEMENT FORMULATION
}

\author{
Bui Thi Thu Hoai ${ }^{1,2, *}$, Nguyen Dinh Kien ${ }^{1,2}$, Tran Thi Thu Huong ${ }^{3}$, Le Thi Ngoc Anh ${ }^{4}$ \\ ${ }^{1}$ Institute of Mechanics, VAST, Hanoi, Vietnam \\ ${ }^{2}$ Graduate University of Science and Technology, VAST, Hanoi, Vietnam \\ ${ }^{3}$ Phenikaa University, Hanoi, Vietnam \\ ${ }^{4}$ Institute of Applied Mechanics and Informatics, VAST, Ho Chi Minh city, Vietnam \\ E-mail: thuhoaihus@gmail.com
}

Received: 18 December 2019 / Published online: 20 March 2020

\begin{abstract}
The large displacements of functionally graded sandwich (FGSW) beams in thermal environment are studied using a finite element formulation. The beams are composed of three layers, a homogeneous core and two functionally graded face sheets with volume fraction of constituents following a power gradation law. The material properties of the beams are considered to be temperature-dependent. Based on Antman beam model and the total Lagrange formulation, a two-node nonlinear beam element taking the effect of temperature rise into account is formulated and employed in the study. The element with explicit expressions for the internal force vector and tangent stiffness matrix is derived using linear interpolations and reduced integration technique to avoid the shear locking. Newton-Raphson based iterative algorithm is employed in combination with the arc-length control method to compute the large displacement response of a cantilever FGSW beam subjected to end forces. The accuracy of the formulated element is confirmed through a comparison study. The effects of the material inhomogeneity, temperature rise and layer thickness ratio on the large deflection response of the beam are examined and highlighted.
\end{abstract}

Keywords: FGSW beam, total Lagrange formulation, reduced integration, thermal environment, large deflection analysis.

\section{INTRODUCTION}

Large displacement analysis of structures has drawn much attention from researchers since the recent invention of new materials allows structures to undergo large deformation during their service. The finite element method, a powerful tool in solving nonlinear problems, is a preferable choice in dealing with this problem. In the context of finite element analysis, two types of nonlinear formulation for analyzing beams undergoing large displacement, namely the co-rotational formulation [1,2] and the total Lagrange

(C) 2020 Vietnam Academy of Science and Technology 
one $[3,4]$, are the most often used. The main difference between these two formulations is the choice of reference frames, which leads to different expressions of the element formulation.

Functionally graded materials (FGMs), a new type of composites initiated by Japanese scientists in mid-1980 [5], are increasing used to fabricate structural elements for use in severe environment. Investigations on nonlinear behaviour of FGM beam structure have been extensively reported in the last two decades. In this line of works, Kang and $\mathrm{Li}[6,7]$ derived the large displacement solutions for cantilever FGM beams subjected to a transverse tip load or a tip moment. The position of the neutral axis has been taken into account in the derivation, which eliminates the axial deformation and bending coupling effect. Kocatürk et al. [8] formulated a total Lagrange formulation for studying large displacement behaviour of FGM beams due to distributed load. Also using the total Lagrange formulation, Almeida et al. [9] investigated geometrically nonlinear behaviour of FGM beams under mechanical loads. Levyakov $[10,11]$ adopted the neutral surface as reference plane to derive the elastic solutions for FGM beams under the thermal loading. Based on the third-order shear deformation beam theory, Zhang [12] derived the constitutive equations for studying the nonlinear bending of FGM beams. Nguyen et al. [13-17] derived the co-rotational beam elements for large displacement analysis of FGM beams and frames. The effect of plastic deformation on buckling and nonlinear bending of FGM beams is considered using the finite element method $[18,19]$. A geometrically exact beam model with fully intrinsic formulation is employed by Masjedi et al. [20] to study the large deflection behaviour of functionally graded beams under conservative and nonconservative loading.

With the development of advanced manufacturing methods [21], FGMs can now be incorporated into sandwich construction to improve the performance of structures. Functionally graded sandwich (FGSW) structures can be designed to have a smooth variation of material properties, and this helps to avoid the interface delaminating problem as often seen in the conventional sandwich structures. Several investigations, mainly the vibration and buckling analyses of FGSW beams, have been reported in recent years [22,23]. Nguyen and Tran [24] are the authors who made the first effort in formulating a corotational beam element for large displacement analysis of FGSW beams and frames. The element using the solution of homogeneous nonlinear equilibrium equations to interpolate displacements is accurate and fast convergence.

In the present work, the large displacement behaviour of FGSW beams in thermal environment is studied by a finite element formulation. The beams considered herein consist of three layers, a homogeneous core and two FGM skin layers. The material properties are assumed to be temperature dependent, and they are graded in the thickness direction by a power gradation law. Based on Antman beam model, a nonlinear beam element using linear interpolation is formulated in the context of the total Lagrange formulation. In order to avoid the shear locking, reduced integration technique is employed to evaluate the strain energy. Numerical investigations are carried to show the accuracy of the formulated element and highlight the influence of the material inhomogeneity, temperature rise and layer thickness ratio on the large displacement behaviour of the beams. 


\section{FGSW BEAM}

An FGSW beam with length $L$, rectangular cross section $(b \times h)$ in a Cartesian coordinate system $(x, z)$ as depicted in Fig. 1 is considered. The beam consists of three layers, a homogeneous isotropic core and two FGM skin layers. The system $(x, z)$ is chosen such that the $x$-axis is on the mid-plane, while the $z$-axis directs upward. Denoting $z_{0}, z_{1}, z_{2}$ and $z_{3}$ are, respectively, the vertical coordinates of the bottom surface, two interfaces between the layers, and the top surface.

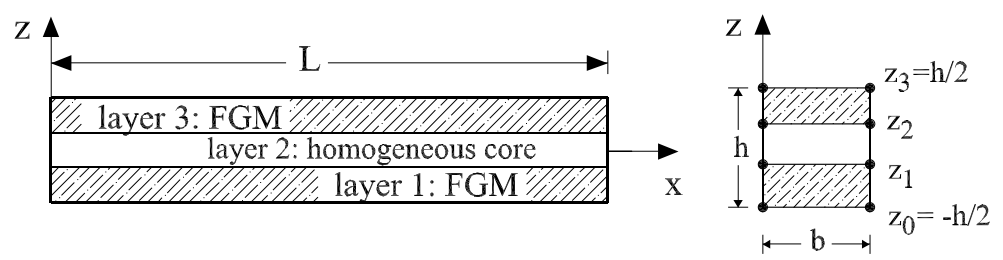

Fig. 1. Geometry and coordinates of an FGSW beam

The beam is assumed forming from two constituent materials, M1 and M2, in which the volume fraction $V_{2}^{(k)}(k=1, \ldots, 3)$ of M2 in the $k^{\text {th }}$ layer varies in the thickness direction according to

$$
\begin{cases}V_{2}^{(1)}=\left(\frac{z_{1}-z}{z_{1}-z_{0}}\right)^{n}, & \text { for } z \in\left[z_{0}, z_{1}\right] \\ V_{2}^{(2)}=0, & \text { for } z \in\left[z_{1}, z_{2}\right] \\ V_{2}^{(3)}=\left(\frac{z_{2}-z}{z_{2}-z_{3}}\right)^{n}, & \text { for } z \in\left[z_{2}, z_{3}\right]\end{cases}
$$

and $V_{1}^{(k)}=1-V_{2}^{(k)}$ is the volume fraction of M1, and $n$ is a non-negative material grading index.

The beam is considered in thermal environment, where significant change in mechanical properties of the constituents is expected. A typical material property $(P)$ depends on the environmental temperature according to [25]

$$
P=P_{0}\left(P_{-1} T^{-1}+1+P_{1} T+P_{2} T^{2}+P_{3} T^{3}\right),
$$

where $P_{0}, P_{-1}, P_{1}, P_{2}$ and $P_{3}$ are the coefficients of temperature $T(\mathrm{~K})$, and they are unique to the constituent materials.

The effective material properties $P_{f}^{(k)}$, like Young's modulus $E_{f}$, thermal expansion coefficient $\alpha_{f}$, and thermal conductivity $\kappa_{f}$, of the $k^{\text {th }}$ layer evaluated by Voigt's model are of the form

$$
P_{f}^{(k)}=P_{1} V_{1}^{(k)}+P_{2} V_{2}^{(k)},
$$

where $P_{1}$ and $P_{2}$ represent the temperature-dependent properties of the M1 and M2, respectively. 
From Eqs. (1) and (3), the effective Young's modulus, thermal expansion coefficient and thermal conductivity can be written in the forms

$$
\begin{aligned}
& E_{f}^{(k)}(z, T)=\left[E_{1}(T)-E_{2}(T)\right] V_{1}^{(k)}+E_{2}(T), \\
& \left.\alpha_{f}^{(k)}(z, T)=\left[\alpha_{1}^{(} T\right)-\alpha_{2}(T)\right] V_{1}^{(k)}+\alpha_{2}(T), \\
& \kappa_{f}^{(k)}(z, T)=\left[\kappa_{1}(T)-\kappa_{2}(T)\right] V_{1}^{(k)}+\kappa_{2}(T),
\end{aligned}
$$

Noting that Poisson's ratio is hardly changed with temperature, and its effective property is simply estimated from values of the constituents by Voigt's model.

\section{FINITE ELEMENT FORMULATION}

A simple two-node beam element for large deflection analysis of FGSW beams in thermal environment is derived in the context the total Lagrange formulation in this section. The element vector of degrees of freedom $(\mathbf{d})$ contains six components as

$$
\mathbf{d}=\left\{\begin{array}{llllll}
u_{1} & w_{1} & \theta_{1} & u_{2} & w_{2} & \theta_{2}
\end{array}\right\}^{T}
$$

where $u_{i}, w_{i}$ and $\theta_{i}(i=1,2)$ are, respectively, the axial, transverse displacements and rotation at node $i$; the superscript ' $T$ ' in Eq. (5) and hereafter, is used to denote the transpose of a vector or a matrix.

The beam element based on Antman beam model [26], originally derived by Pacoste and Eriksson [27], has been employed by Nguyen [4], Almeida et al. [9] in nonlinear analysis of beams. Fig. 2 shows the initial and deformed configurations of a two-node beam element with length of $l$ in a Cartesian coordinate system $(x, z)$. The deformation at a point with initial abscissa $x$, measured from the left node, can be defined by mean of the angle $\theta(x)$ - the rotation of the cross section $S$ associated with the point, and the position vector $\mathbf{r}(x)$ defined as [28]

$$
\mathbf{r}(x)=[x+u(x)] \mathbf{i}+w(x) \mathbf{j},
$$

where $\mathbf{i}$ and $\mathbf{j}$ are, respectively, the base unit vectors of the $x$ - and $z$-axes; $0 \leq x \leq l$ is measured on the initial configuration; $u(x)$ and $w(x)$ are the axial and transverse displacements of the point on the $x$-axis.

The cross section $S$ associated with the point, as depicted in Fig. 2, may undergo large displacement and rotation according to displacements $u(x), w(x)$ and rotation $\theta(x)$. The vector $\mathbf{r}^{\prime}(x)$ tangent to the deformed beam can be expressed in terms of strain measures as

$$
\mathbf{r}^{\prime}(x)=\frac{\partial \mathbf{r}(x)}{\partial x}=[1+\epsilon(x)] \mathbf{e}_{1}+\gamma(x) \mathbf{e}_{2}, \quad \kappa(x)=\frac{\partial \theta(x)}{\partial x}
$$

where

$$
\mathbf{e}_{1}=\cos \theta \mathbf{i}+\sin \theta \mathbf{j}, \quad \mathbf{e}_{2}=-\sin \theta \mathbf{i}+\cos \theta \mathbf{j},
$$


are, respectively, the unit vectors, orthogonal and parallel to the current cross section; $\epsilon(x)$ and $\gamma(x)$ are, respectively, the axial and shear strains, which with the help of Eqs. (6)(8) can be written in the forms

$$
\begin{aligned}
& \epsilon(x)=\left(1+\frac{\partial u}{\partial x}\right) \cos \theta+\frac{\partial w}{\partial x} \sin \theta-1, \\
& \gamma(x)=\frac{\partial w}{\partial x} \cos \theta-\left(1+\frac{\partial u}{\partial x}\right) \sin \theta .
\end{aligned}
$$

Noting that the above axial strain $\epsilon(x)$, shear strain $\gamma(x)$ and curvature $\kappa(x)$, as emphasized in [27], although parameterized for convenience by the reference abscissa $x \in[0, l]$ take the values on the current deformed configuration.

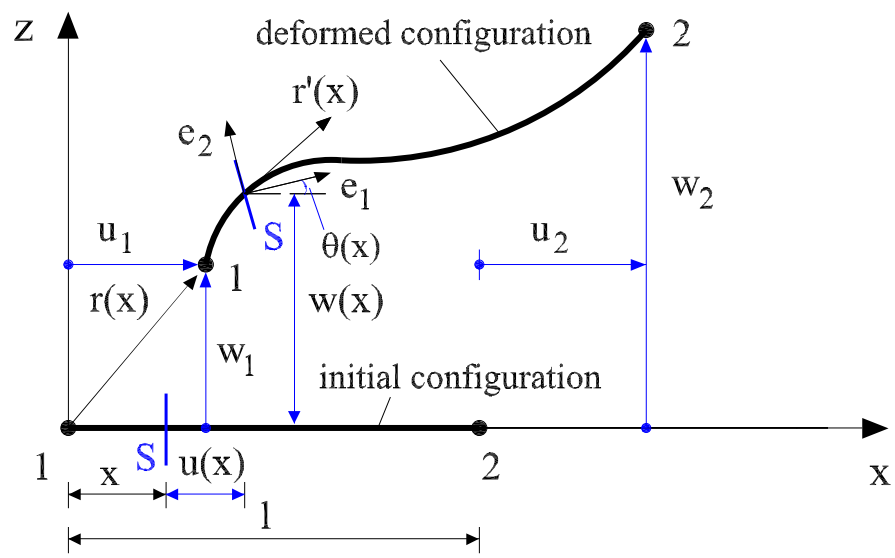

Fig. 2. Configurations and kinematics of beam element

The strain energy for the shear deformable beam element is of the form

$$
U_{B}=\frac{1}{2} \int_{0}^{l}\left[A_{11} \epsilon(x)^{2}+2 A_{12} \epsilon(x) \kappa(x)+A_{22} \kappa(x)^{2}+\psi A_{33} \gamma(x)^{2}\right] \mathrm{d} x,
$$

where $\psi$ is the shear correction factor, chosen by $5 / 6$ for the rectangular cross section; $A_{11}, A_{12}, A_{22}$ and $A_{33}$ are, respectively, the axial, axial-bending coupling, bending and shear rigidities, which are defined as

$$
\begin{aligned}
\left(A_{11}, A_{12}, A_{22}\right) & =\int_{A} E_{f}^{(k)}\left(1, z, z^{2}\right) \mathrm{d} A=\sum_{k=1}^{3} \int_{z_{k-1}}^{z_{k}} b E_{f}^{(k)}\left(1, z, z^{2}\right) \mathrm{d} z, \\
A_{33} & =\int_{A} G_{f}^{(k)} \mathrm{d} A=\sum_{k=1}^{3} \int_{z_{k-1}}^{z_{k}} b G_{f}^{(k)} \mathrm{d} z
\end{aligned}
$$


with $A$ is the cross-sectional area. Noting that both $E_{f}^{(k)}$ and $G_{f}^{(k)}$ in Eq. (11) are the temperature-dependent effective moduli.

Suppose the beam is initially stress free at temperature $T_{0}$. The beam is initially stressed by the temperature rise. The initial stress due to temperature rise is

$$
\sigma_{x T}^{(k)}=-E_{f}^{(k)}(z, T) \alpha_{f}^{(k)}(z, T) \Delta T,
$$

where the effective Young's modulus $E_{f}^{(k)}(z, T)$ and thermal expansion coefficient $\alpha_{f}^{(k)}(z, T)$ are given by Eq. (4); $\Delta T=T-T_{0}$ is the temperature rise, assume to be uniform for the present work.

The strain energy resulted from the temperature rise is of the form [29]

$$
U_{T}=\frac{1}{2} \int_{0}^{l} N_{T}\left(\frac{\partial w(x)}{\partial x}\right)^{2} \mathrm{~d} x,
$$

with $N_{T}$ is the axial force caused by the elevated temperature, defined as

$$
N_{T}=\int_{A} \sigma_{x T}^{(k)} \mathrm{d} A=-\sum_{k=1}^{3} b \int_{z_{k-1}}^{z_{k}} E_{f}^{(k)}(z, T) \alpha_{f}^{(k)}(z, T) \Delta T \mathrm{~d} z .
$$

As the shear deformation is taken into account, the transverse displacement $w(x)$ is independent of the rotation $\theta(x)$, and linear functions can be employed to interpolate the displacements and rotation as

$$
u=\frac{l-x}{l} u_{1}+\frac{x}{l} u_{2}, w=\frac{l-x}{l} w_{1}+\frac{x}{l} w_{2}, \theta=\frac{l-x}{l} \theta_{1}+\frac{x}{l} \theta_{2} .
$$

The beam element based on the above linear interpolation functions, however encounters the shear locking problem [30]. To overcome this problem, one-point Gauss quadrature is used herewith to evaluate the strain energy of the beam element. In this regards and using Eq. (15), one can write the strain energy due to the beam deformation, Eq. (10), in the form

$$
U_{B}=\frac{l}{2}\left(A_{11} \bar{\varepsilon}^{2}+2 A_{12} \bar{\varepsilon} \overline{\mathcal{K}}+A_{22} \bar{\kappa}^{2}+\psi A_{33} \bar{\gamma}^{2}\right),
$$

and also the strain energy (13) due to the temperature rise as

$$
U_{T}=\frac{l}{2} N_{T}\left(\frac{w_{2}-w_{1}}{l}\right)^{2}
$$

In Eq. (16), $\bar{\varepsilon}, \bar{\gamma}$ and $\bar{\kappa}$ are given by

$$
\left\{\begin{array}{l}
\bar{\varepsilon}=\left(1+\frac{u_{2}-u_{1}}{l}\right) \cos \bar{\theta}+\frac{w_{2}-w_{1}}{l} \sin \bar{\theta}-1, \\
\bar{\gamma}=-\left(1+\frac{u_{2}-u_{1}}{l}\right) \sin \bar{\theta}+\frac{w_{2}-w_{1}}{l} \cos \bar{\theta}, \\
\bar{\kappa}=\frac{\theta_{2}-\theta_{1}}{l}, \quad \text { with } \bar{\theta}=\frac{\theta_{1}+\theta_{2}}{2} .
\end{array}\right.
$$


The internal force vector $\mathbf{f}_{i n}$ and tangent stiffness matrix $\mathbf{k}_{t}$ for the element are obtained by one and twice differentiating the total strain energy, $U=U_{B}+U_{T}$, resulted from the beam deformation and the temperature rise with respect to the nodal degrees of freedom as

$$
\begin{aligned}
\mathbf{f}_{i n} & =\frac{\partial U}{\partial \mathbf{d}}=\mathbf{f}_{a}+\mathbf{f}_{c}+\mathbf{f}_{b}+\mathbf{f}_{s}+\mathbf{f}_{T}, \\
\mathbf{k}_{t} & =\frac{\partial^{2} U}{\partial \mathbf{d}^{2}}=\mathbf{k}_{a}+\mathbf{k}_{c}+\mathbf{k}_{b}+\mathbf{k}_{s}+\mathbf{k}_{T},
\end{aligned}
$$

where the subscripts $a, c, b, s, T$ denote the terms stemming from the axial stretching, axial-bending coupling, bending, shear deformation of the beam and the temperature rise, respectively.

Noting that for the nonlinear analysis considered herein, both the internal force vector $\mathbf{f}_{i n}$ and the tangent stiffness matrix $\mathbf{k}_{t}$ depend on the current nodal displacements d. The detailed expressions for the internal force vector and tangent stiffness matrix in Eq. (19) are given by Eqs. (23)-(29) in the Appendix.

\section{EQUILIBRIUM EQUATION}

The equilibrium equation for large deflection analysis of the beam can be written in the form [31]

$$
\mathbf{g}(\mathbf{p}, \lambda)=\mathbf{q}_{\text {in }}(\mathbf{p})-\lambda \mathbf{f}_{e x}=\mathbf{0},
$$

where the residual force vector $\mathbf{g}$ is a function of the current structural nodal displacements $\mathbf{p}$ and the load level parameter $\lambda ; \mathbf{q}_{\text {in }}$ is the structural nodal force vector, assembled from the formulated vector $\mathbf{f}_{i n} ; \mathbf{f}_{e x}$ is the fixed external loading vector.

The system of Eq. (20) can be solved by an incremental/iterative procedure. The procedure results in a predictor-corrector algorithm, in which a new solution is firstly predicted from a previous converged solution, and then successive corrections are added until a chosen convergence criterion is satisfied. A convergence criterion based on Euclidean norm of the residual force vector is used herein as

$$
\|\mathbf{g}\|=<\epsilon\left\|\lambda \mathbf{f}_{e x}\right\|
$$

where $\epsilon$ is the tolerance, chosen by $10^{-4}$ for all numerical examples reported in Section 5 .

Newton-Raphson based method is used in combination with the spherical arc-length control technique herein to solve Eq. (20). Detail implementation of the spherical arclength control method is given in [31].

\section{NUMERICAL INVESTIGATION}

Numerical investigation is carried out in this section to show the accuracy of the derived beam formulation and to illustrate the effects of the beam parameters and temperature rise on the large displacement behaviour of the FGSW beam. To this end, a cantilever beam made of stainless steel (SUS304 - M1) and Silicon Nitride $\left(\mathrm{Si}_{3} \mathrm{~N}_{4}-\mathrm{M} 2\right)$ with the core is pure M1, under a tip load $P$ and a tip moment $M$ is considered. The temperature-dependent coefficients for the constituent materials of the beam are listed in Tab. 1. A Poison's ratio $v=0.3$ is chosen for both the constituent materials. Otherwise 
stated, an aspect ratio $L / h=10$ is chosen for the analysis. Three numbers in the brackets are used to denote the layer thickness ratio, e.g. (2-1-1) means that the thickness ratios of the bottom layer, the core and the top layer is (2:1:1). The following dimensionless parameters are introduced for the external loads and displacements

$$
P^{*}=\frac{P L^{2}}{E_{S} I}, M^{*}=\frac{M L}{E_{S} I}, u^{*}=\frac{u_{L}}{L}, w^{*}=\frac{w_{L}}{L},
$$

where $I$ is the inertia moment of the cross section; $E_{s}$ is Young's modulus of steel; $u_{L}$ and $w_{L}$ are the tip axial and transverse displacements, respectively.

Table 1. Temperature-dependent coefficients for constituent materials [32]

\begin{tabular}{ccccccc}
\hline Material & Property & $P_{0}$ & $P_{-1}$ & $P_{1}$ & $P_{2}$ & $P_{3}$ \\
\hline \multirow{2}{*}{$\mathrm{Si}_{3} \mathrm{~N}_{4}$} & $\mathrm{E}(\mathrm{Pa})$ & $348.43 \times 10^{9}$ & 0.0 & $-3.07 \times 10^{-4}$ & $2.16 \times 10^{-7}$ & $-8.946 \times 10^{-11}$ \\
& $\alpha(1 / \mathrm{K})$ & $5.8723 \times 10^{-6}$ & 0.0 & $9.095 \times 10^{-4}$ & 0.0 & 0.0 \\
& $\kappa(\mathrm{W} / \mathrm{mK})$ & 13.723 & 0.0 & $-1.032 \times 10^{-3}$ & $5.466 \times 10^{-7}$ & $-7.876 \times 10^{-11}$ \\
\hline \multirow{2}{*}{$\mathrm{SUS304}$} & $\mathrm{E}(\mathrm{Pa})$ & $201.04 \times 10^{9}$ & 0.0 & $3.079 \times 10^{-4}$ & $-6.534 \times 10^{-7}$ & 0.0 \\
& $\alpha(1 / \mathrm{K})$ & $12.33 \times 10^{-6}$ & 0.0 & $8.086 \times 10^{-4}$ & 0.0 & 0.0 \\
& $\kappa(\mathrm{W} / \mathrm{mK})$ & 15.379 & 0.0 & $-1.264 \times 10^{-3}$ & $2.092 \times 10^{-6}$ & $-7.223 \times 10^{-10}$ \\
\hline
\end{tabular}

\subsection{Accuracy and convergence studies}

Firstly, the accuracy and convergence of the derived beam element are necessary to verify. To this end, Fig. 3 compares the tip response of a cantilever FGSW beam under a transverse tip load of the present work with the result of Ref. [24] using a co-rotational formulation. The result in Fig. 3 is obtained for the beam formed from Aluminum and
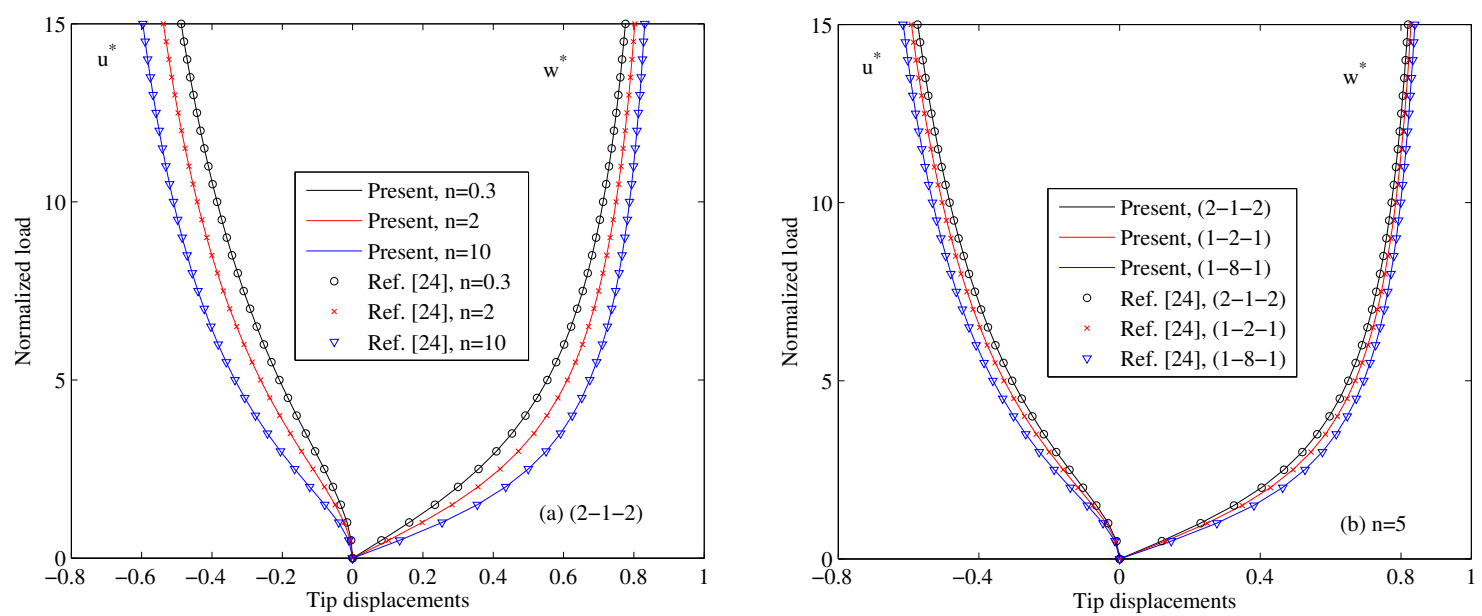

Fig. 3. Comparison of tip response of cantilever FGSW beam under a transverse tip load 
Zirconia with the material and geometric data given in [24]. Very good agreement between the result of the present work and that of Ref. [24] is noted from Fig. 3, regardless of the material grading index and the layer thickness ratio.

The convergence of the element is shown in Tab. 2, where the dimensionless deflections of the (2-1-2) and (2-2-1) cantilever beams under a tip transverse load $P^{*}=10$ obtained by different number of the elements are given for $\Delta T=40 \mathrm{~K}$ and various values of the grading index. As seen from Tab. 2, the convergence of the element can be achieved by using twenty elements, regardless of the material grading indexes and the thickness ratio. In this regard, a mesh of twenty elements is used in all the computations reported below.

Table 2. Convergence of the element in evaluating dimensionless deflection $w^{*}$ of cantilever FMSW beam under a tip transverse load $\left(P^{*}=10, \Delta T=40 \mathrm{~K}\right)$

\begin{tabular}{|c|c|c|c|c|c|c|c|c|}
\hline \multirow{2}{*}{ nELE } & \multicolumn{4}{|c|}{$(2-1-2)$} & \multicolumn{4}{|c|}{$(2-2-1)$} \\
\hline & $n=0.3$ & $n=0.5$ & $n=1$ & $n=5$ & $n=0.3$ & $n=0.5$ & $n=1$ & $n=5$ \\
\hline 6 & 0.7805 & 0.7841 & 0.7911 & 0.8115 & 0.7892 & 0.7928 & 0.7993 & 0.8162 \\
\hline 8 & 0.7810 & 0.7846 & 0.7916 & 0.8121 & 0.7897 & 0.7933 & 0.7998 & 0.8167 \\
\hline 10 & 0.7812 & 0.7849 & 0.7918 & 0.8123 & 0.7899 & 0.7935 & 0.8000 & 0.8170 \\
\hline 12 & 0.7813 & 0.7850 & 0.7919 & 0.8124 & 0.7901 & 0.7937 & 0.8001 & 0.8171 \\
\hline 14 & 0.7814 & 0.7851 & 0.7920 & 0.8125 & 0.7901 & 0.7938 & 0.8002 & 0.8172 \\
\hline 16 & 0.7815 & 0.7851 & 0.7921 & 0.8126 & 0.7902 & 0.7938 & 0.8003 & 0.8173 \\
\hline 18 & 0.7815 & 0.7852 & 0.7921 & 0.8126 & 0.7902 & 0.7938 & 0.8003 & 0.8173 \\
\hline 20 & 0.7815 & 0.7852 & 0.7921 & 0.8126 & 0.7902 & 0.7938 & 0.8003 & 0.8173 \\
\hline
\end{tabular}

\subsection{Cantilever FGSW beam under a transverse tip load}

A cantilever FGSW beam in thermal environment under a transverse tip load $P$ is considered in this subsection. The dimensionless tip deflections of the beam corresponding to a transverse tip load $P^{*}=10$ are listed in Tab. 3 for different values of the index $n$, the layer thickness ratio and the temperature rise. The effect of the material distribution and the temperature rise is clearly seen from Tab. 3, where the deflection is seen to be increased by the increase of the grading index and the temperature rise, regardless of the layer thickness ratio. The increase of the deflection by increasing the index $n$ can be explained by the higher content of SUS304 for the beam associated with a higher index $n$, as seen from Eq. (1). Since Young's modulus of SUS304 is lower than that of $\mathrm{Si}_{3} \mathrm{~N}_{4}$, and thus the rigidities of the beam with a higher index $n$ are lower, and this leads to a higher deflection. The increase of the deflection for the beam subjected to the higher temperature rise is resulted from the decrease of the Young's modulus and the increase of the axial force $N_{T}$. The effect of the force $N_{T}$ is similar to that of an axial compressive force, which causes the decrease of the bending rigidity. The influence of the layer thickness 
ratio on the tip deflection in Tab. 3 can also be explained by the change in the rigidities of the beam.

Table 3. Tip deflection $w^{*}$ of cantilever beam in thermal environment corresponding to a tip load $P^{*}=10$

\begin{tabular}{clllllll}
\hline$\Delta T(\mathrm{~K})$ & $n$ & $(1-0-1)$ & $(2-1-2)$ & $(2-1-1)$ & $(2-2-1)$ & $(1-3-1)$ & $(1-8-1)$ \\
\hline \multirow{3}{*}{0} & 0.3 & 0.7708 & 0.7732 & 0.7780 & 0.7821 & 0.7868 & 0.8013 \\
& 0.5 & 0.7739 & 0.7769 & 0.7816 & 0.7867 & 0.7906 & 0.8039 \\
& 1 & 0.7802 & 0.7823 & 0.7965 & 0.7923 & 0.7973 & 0.8084 \\
& 5 & 0.8018 & 0.8051 & 0.8074 & 0.8100 & 0.8132 & 0.8181 \\
\hline \multirow{3}{*}{30} & 0.3 & 0.7769 & 0.7795 & 0.7842 & 0.7882 & 0.7930 & 0.8070 \\
& 0.5 & 0.7801 & 0.7813 & 0.7878 & 0.7919 & 0.7967 & 0.8096 \\
& 1 & 0.7864 & 0.7901 & 0.7944 & 0.7984 & 0.8032 & 0.8139 \\
& 5 & 0.8076 & 0.8108 & 0.8130 & 0.8155 & 0.8186 & 0.8233 \\
\hline \multirow{3}{*}{50} & 0.3 & 0.7809 & 0.7835 & 0.7882 & 0.7922 & 0.7969 & 0.8108 \\
& 0.5 & 0.7841 & 0.7872 & 0.7918 & 0.7958 & 0.8006 & 0.8133 \\
& 1 & 0.7904 & 0.7941 & 0.7984 & 0.8023 & 0.8070 & 0.8175 \\
& 5 & 0.8114 & 0.8145 & 0.8167 & 0.8191 & 0.8221 & 0.8267 \\
\hline \multirow{3}{*}{90} & 0.3 & 0.7885 & 0.7913 & 0.7959 & 0.7999 & 0.8046 & 0.8181 \\
& 0.5 & 0.7918 & 0.7950 & 0.7995 & 0.8035 & 0.8082 & 0.8205 \\
& 1 & 0.7982 & 0.8019 & 0.8061 & 0.8099 & 0.8144 & 0.8246 \\
& 5 & 0.8186 & 0.8217 & 0.8238 & 0.8262 & 0.8290 & 0.8334 \\
\hline
\end{tabular}

The effect of the temperature rise and the layer thickness ratio on the large displacement response of the FGSW beam can also be seen from Figs. 4 and 5, where the loaddisplacement curves of the FGSW beam are shown for various values of the temperature rise and the layer thickness ratio. At a given value of the applied load, the tip displacements increase as the temperature rise $\Delta T$ increases. The tip displacements of the beam, as seen from Fig. 5, are also increased by the increase of the core thickness, regardless of the load level and the temperature rise. The increase of the displacements, as explained above, is resulted from the lower rigidities of the beam associated with a larger core thickness. The deformed configurations of the beam corresponding to an applied transverse tip load $P^{*}=5$ as depicted in Fig. 6 also confirm the effects of the temperature rise and the layer thickness ratio on the large displacement response of the FGSW beam.

In Figs. 7 and 8, the thickness distribution of the axial stress on the clamped end section of the FGSW cantilever beam under the transverse tip load is depicted for a transverse load $P^{*}=3$ and various values of the temperature rise and the layer thickness ratio. Different from homogeneous and functionally graded beams, the curves for stress distribution of the FGSW beam consist of three distinct parts, in which the stress distribution 

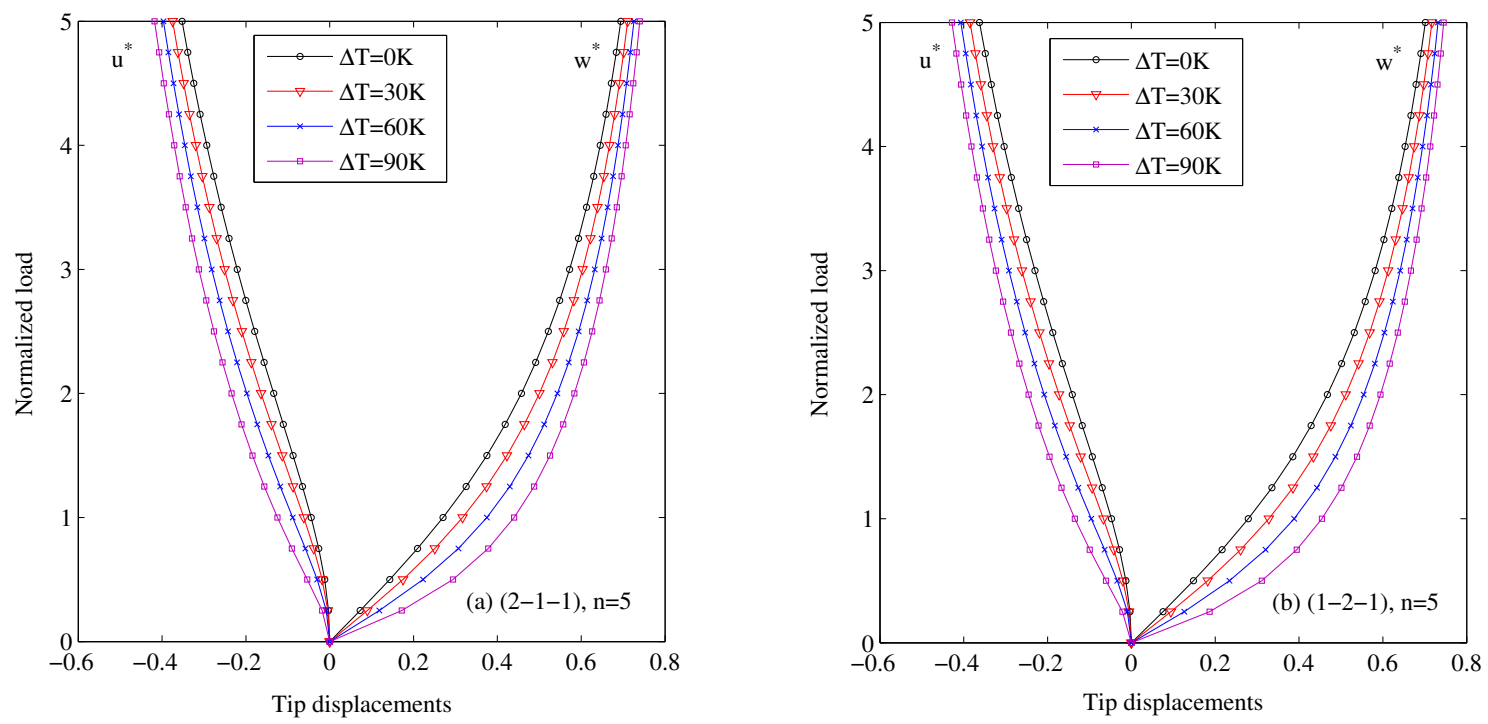

Fig. 4. Effect of temperature rise on large displacement response of the FGSW beam under a transverse tip load
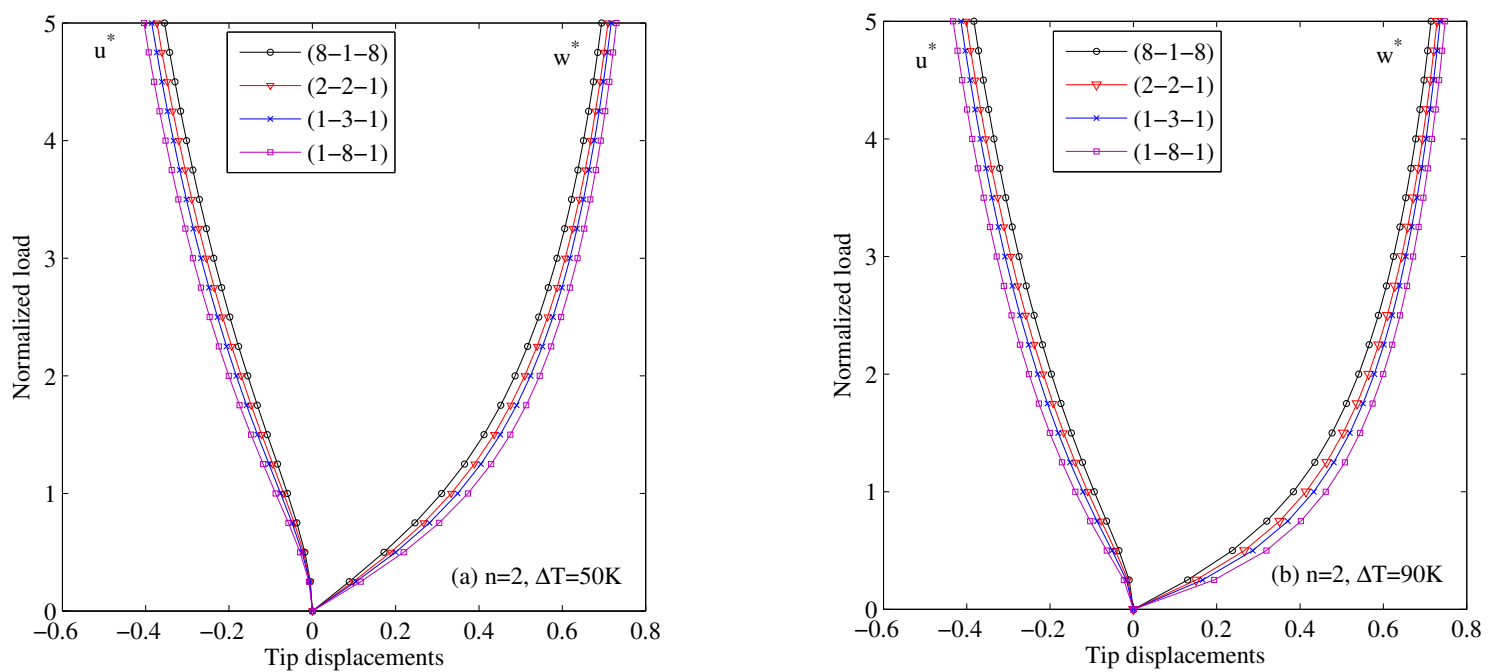

Fig. 5. Effect of layer thickness ratio on large displacement response of the FGSW beam under a transverse tip load

in the two functionally graded layers is not linear due to the power-law variation of the effective modulus. The temperature rise, as seen from Fig. 7, alters the axial stress, and the maximum stress increases by the increase of the temperature rise. The influence of the core thickness to the axial stress, as seen from Fig. 8 is similar to that of the temperature rise, and the maximum stress is higher for the beam with a larger core thickness. 

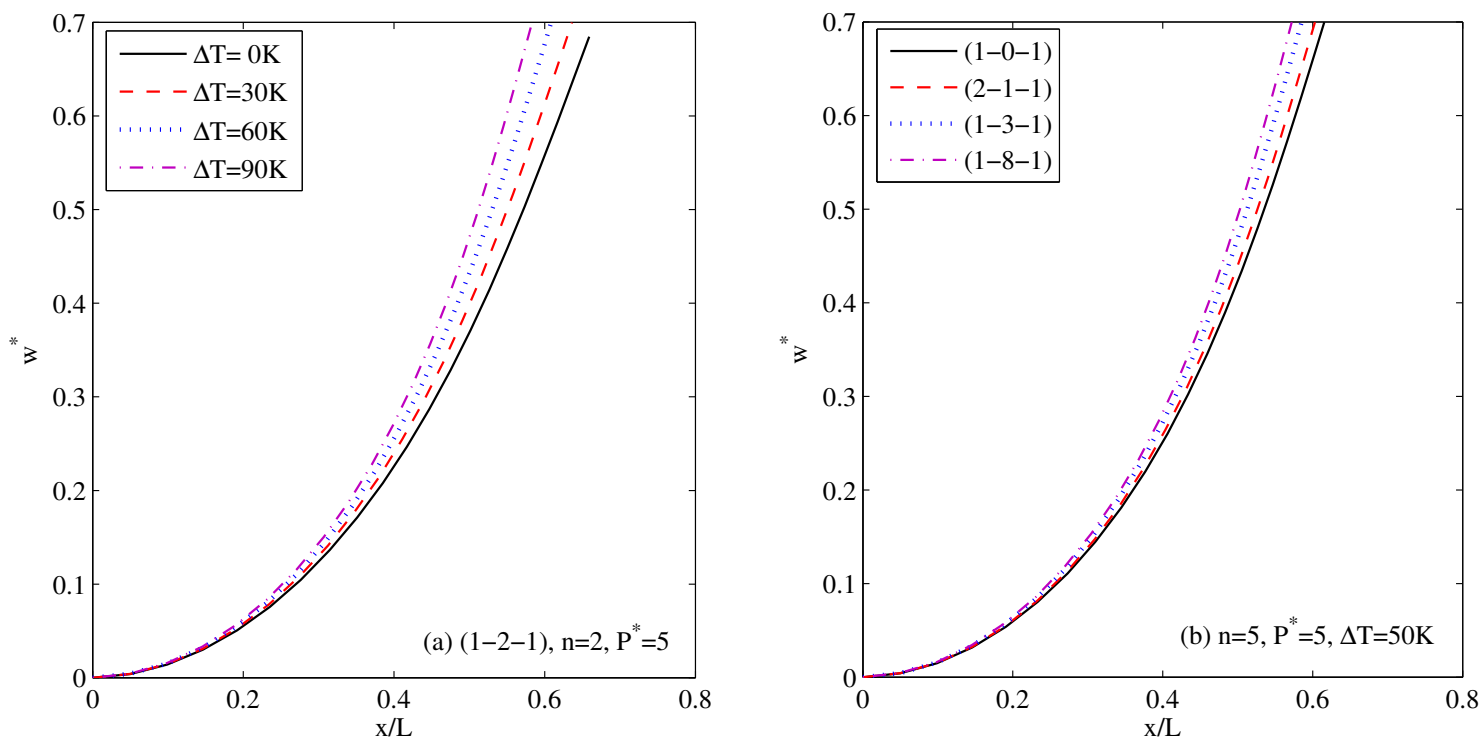

Fig. 6. Deformed configurations of cantilever FGSW beam corresponding to a transverse tip load $P^{*}=5$
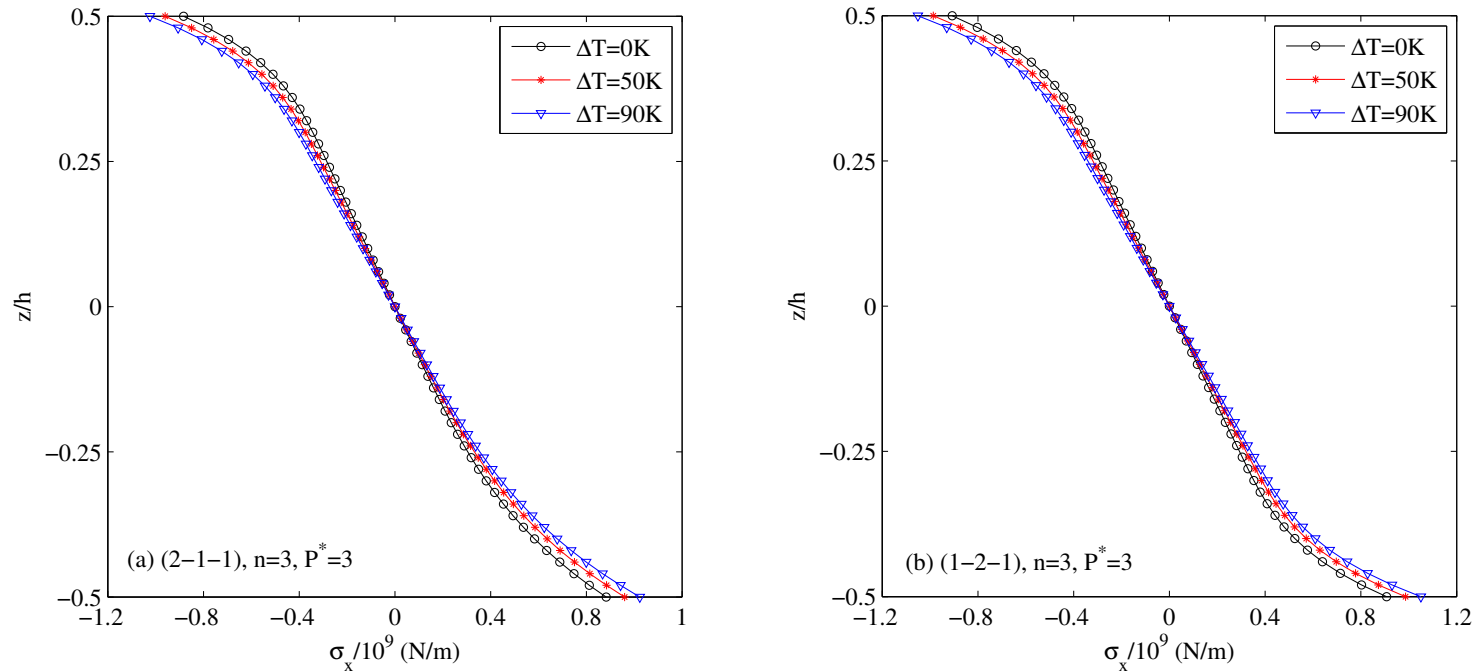

Fig. 7. Effect of temperature rise on thickness distribution of axial stress at clamped section of FGSW cantilever beam corresponding to a transverse tip load $P^{*}=3$ 

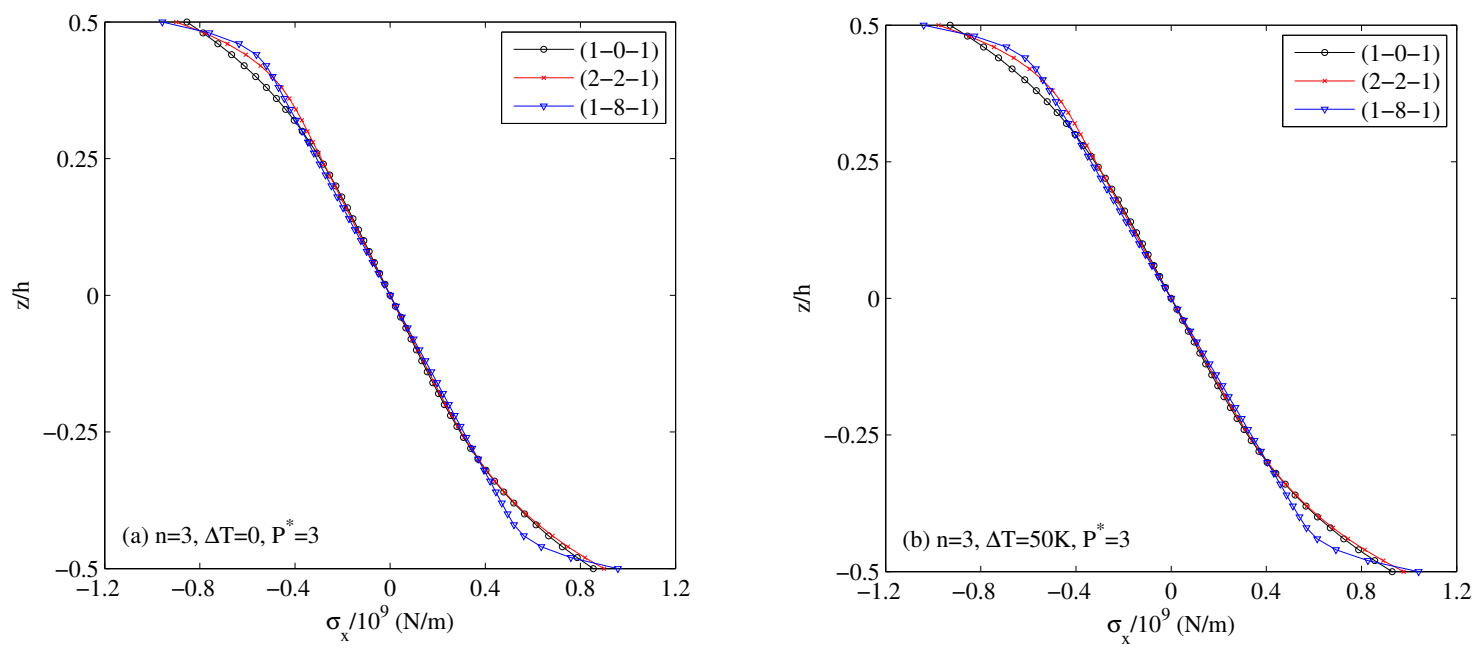

Fig. 8. Effect of layer thickness ratio on thickness distribution of axial stress at clamped section of FGSW cantilever beam corresponding to a transverse tip load $P^{*}=3$

\subsection{Roll-up of cantilever beam due to a tip moment}

The roll-up of a cantilever FGSW beam subjected to a tip moment $M$ is studied in this sub-section. In Figs. 9 and 10, the equilibrium paths of the beam are respectively depicted for different values of the temperature rise and the layer thickness ratio. The temperature rise and the layer thickness ratio, as seen from the figures, play an important role on the large displacement behaviour of the beam. The effect of the layer thickness ratio on the response of the beam is more significant in the large displacement region than that of
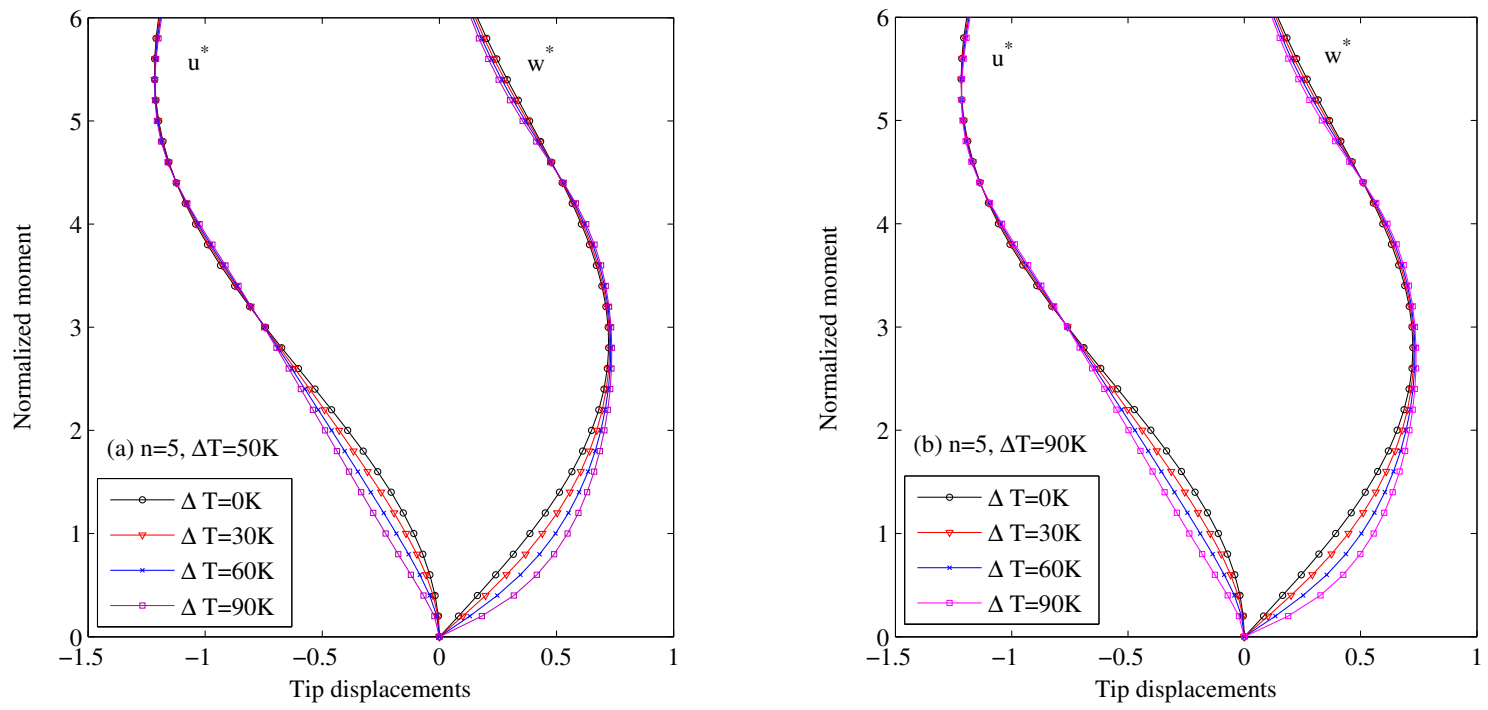

Fig. 9. Equilibrium paths of cantilever FGSW beam under a tip moment for different temperature rise 

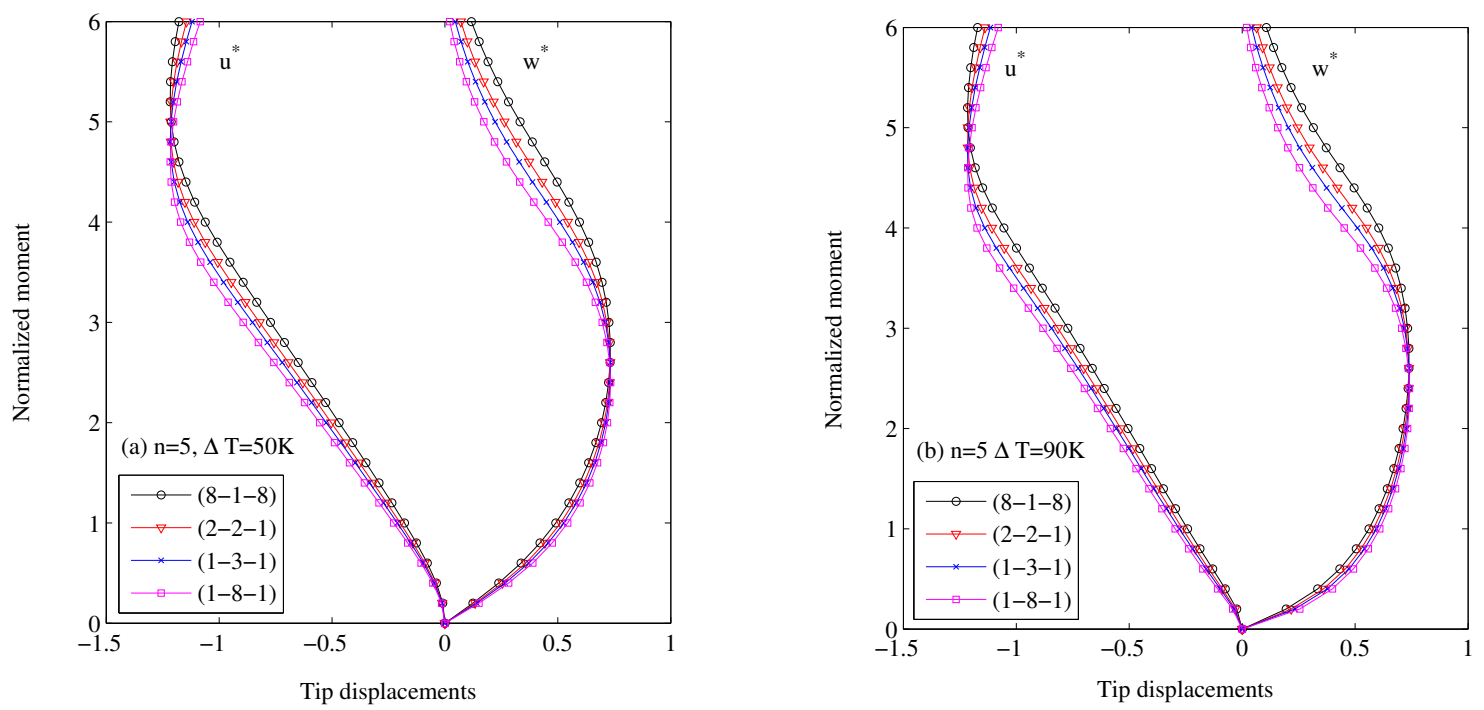

Fig. 10. Equilibrium paths of cantilever FGSW beam under a tip moment for different layer thickness ratio
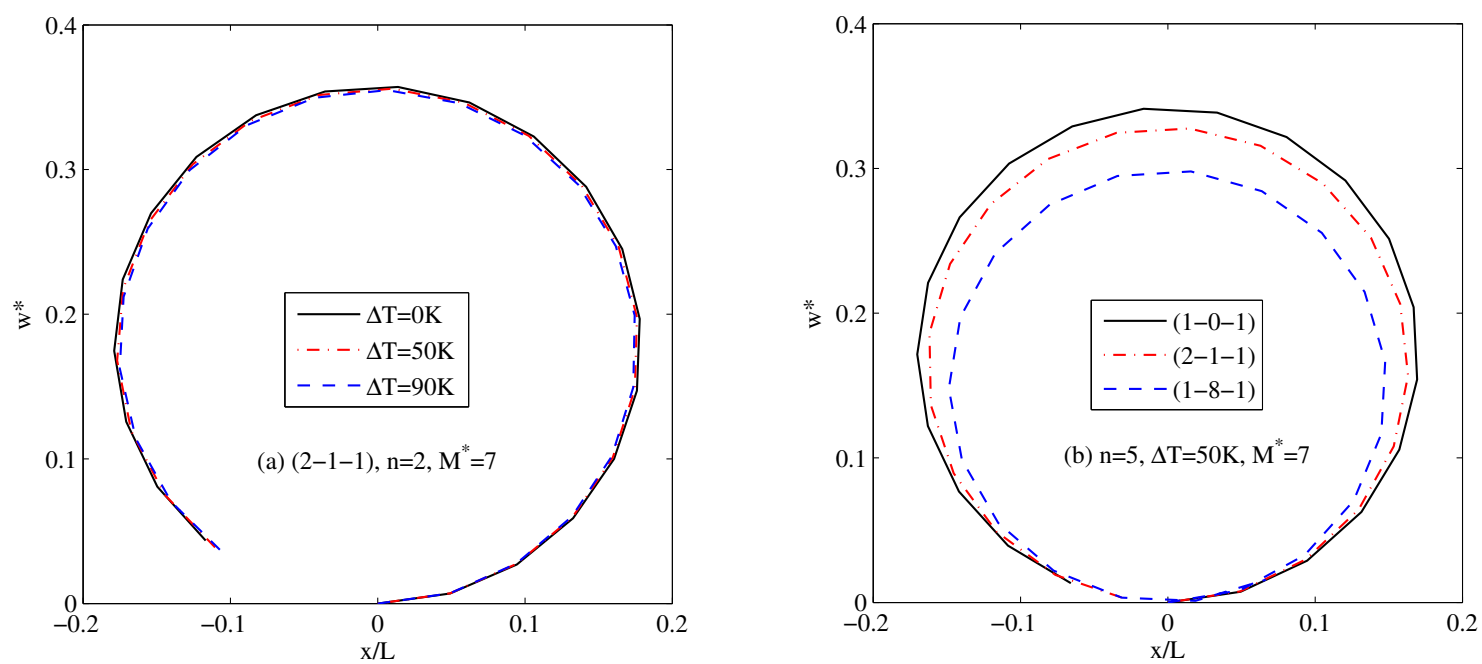

Fig. 11. Deformed configurations of FGSW beam subjected to a tip moment

the temperature rise. The significant influence of the layer thickness ratio on the large displacement behaviour of the FGSW beam can be seen more clearly from Fig. 11, where the deformed configurations of the beam are displayed for $M^{*}=7$ and different values of the temperature rise and layer thickness ratio. At the applied moment $M^{*}=7$, the (1-8-1) beam has already rolled up to a circle while the (1-0-1) beam has not yet. Noting that due to the snap-back of the equilibrium paths, the arc-length control method must be employed to trace the paths in Figs. 9 and 10. 


\section{CONCLUSIONS}

The large displacement behaviour of FGSW beams in thermal environment has been investigated by a finite element formulation. The beams are considered to be composed of three layers, a homogeneous core and two functionally graded skin layers with the temperature-dependent material properties. Based on the Antman beam model, a firstorder shear deformable nonlinear beam element taking the effect of temperature rise into account was formulated in the context of the total Lagrange formulation. The element with explicit expressions for the internal force vector and tangent stiffness matrix has been derived using the reduced integration technique to avoid the shear locking. Using the derived beam element, the large displacement response of a cantilever FGSW beam under the end forces has been computed, and the effects of the material inhomogeneity, temperature rise and layer thickness ratio have been examined. The obtained numerical results reveal that, in addition to the material inhomogeneity, the temperature rise and the layer thickness ratio also play an important role on the large displacement behaviour of the beam. It has been shown that the effect of the layer thickness ratio on the behaviour of the FGSW beams in the large displacement region is more significant than that of the temperature rise. It is necessary to note that though the numerical investigation in the present paper has been carried out for the cantilever beam only, the element formulation formulated herein can be used to analyze the FGSW beams with other boundary conditions as well. Additionally, the present beam formulation is simple, and its extension to the large displacement analysis of beams made of other materials, e.g., functionally graded carbon nanotube reinforced composite beams, is straightforward.

\section{ACKNOWLEDGEMENTS}

The work presented in this article was supported by National Foundation for Science and Technology Development (NAFOSTED), grant number 107.02-2018.23 and Vietnam Academy of Science and Technology, grant number QTRU01.07/20-21.

\section{REFERENCES}

[1] D. K. Nguyen. A non-linear element for analysing elastic frame structures at large deflections. Vietnam Journal of Mechanics, 22, (1), (2000), pp. 19-28. https://doi.org/10.15625/08667136/9959.

[2] D. K. Nguyen and Q. Q. Do. Large deflection analysis of frames by elements containing higher-order terms. Vietnam Journal of Mechanics, 25, (4), (2003), pp. 243-254. https://doi.org/10.15625/0866-7136/25/4/6595.

[3] R. D. Wood and O. C. Zienkiewicz. Geometrically nonlinear finite element analysis of beams, frames, arches and axisymmetric shells. Computers \& Structures, 7, (6), (1977), pp. 725-735. https://doi.org/10.1016/0045-7949(77)90027-x.

[4] D. K. Nguyen. Postbuckling behavior of beams on two-parameter elastic foundation. International Journal of Structural Stability and Dynamics, 4, (01), (2004), pp. 21-43. https://doi.org/10.1142/s0219455404001082.

[5] M. Koizumi. FGM activities in Japan. Composites Part B: Engineering, 28, (1-2), (1997), pp. 1-4. https://doi.org/10.1016/s1359-8368(96)00016-9. 
[6] Y. A. Kang and X. F. Li. Bending of functionally graded cantilever beam with power-law non-linearity subjected to an end force. International Journal of Non-Linear Mechanics, 44, (6), (2009), pp. 696-703. https://doi.org/10.1016/j.ijnonlinmec.2009.02.016.

[7] Y. A. Kang and X. F. Li. Large deflections of a non-linear cantilever functionally graded beam. Journal of Reinforced Plastics and Composites, 29, (12), (2010), pp. 1761-1774. https://doi.org/10.1177/0731684409103340.

[8] T. Kocatürk, M. Şimşek, and Ş. D. Akbaş. Large displacement static analysis of a cantilever Timoshenko beam composed of functionally graded material. Science and Engineering of Composite Materials, 18, (1-2), (2011), pp. 21-34. https://doi.org/10.1515/secm.2011.005.

[9] C. A. Almeida, J. C. R. Albino, I. F. M. Menezes, and G. H. Paulino. Geometric nonlinear analyses of functionally graded beams using a tailored Lagrangian formulation. Mechanics Research Communications, 38, (8), (2011), pp. 553-559. https://doi.org/10.1016/j.mechrescom.2011.07.006.

[10] S. V. Levyakov. Elastica solution for thermal bending of a functionally graded beam. Acta Mechanica, 224, (8), (2013), pp. 1731-1740. https://doi.org/10.1007/s00707-013-0834-1.

[11] S. V. Levyakov. Thermal elastica of shear-deformable beam fabricated of functionally graded material. Acta Mechanica, 226, (3), (2015), pp. 723-733. https://doi.org/10.1007/s00707-0141218-x.

[12] D. G. Zhang. Nonlinear bending analysis of FGM beams based on physical neutral surface and high order shear deformation theory. Composite Structures, 100, (2013), pp. 121-126. https://doi.org/10.1016/j.compstruct.2012.12.024.

[13] D. K. Nguyen. Large displacement response of tapered cantilever beams made of axially functionally graded material. Composites Part B: Engineering, 55, (2013), pp. 298-305. https://doi.org/10.1016/j.compositesb.2013.06.024.

[14] D. K. Nguyen. Large displacement behaviour of tapered cantilever Euler-Bernoulli beams made of functionally graded material. Applied Mathematics and Computation, 237, (2014), pp. 340-355. https://doi.org/10.1016/j.amc.2014.03.104.

[15] D. K. Nguyen, T. H. Trinh, and T. H. Le. A co-rotational beam element for geometrically nonlinear analysis of plane frames. Vietnam Journal of Mechanics, 35, (1), (2013), pp. 51-65. https://doi.org/10.15625/0866-7136/35/1/2892.

[16] D. K. Nguyen and B. S. Gan. Large deflections of tapered functionally graded beams subjected to end forces. Applied Mathematical Modelling, 38, (11-12), (2014), pp. 3054-3066.

[17] D. K. Nguyen, B. S. Gan, and T. H. Trinh. Geometrically nonlinear analysis of planar beam and frame structures made of functionally graded material. Structural Engineering and Mechanics, 49, (6), (2014), pp. 727-743. https://doi.org/10.12989/sem.2014.49.6.727.

[18] T. H. Trinh, D. K. Nguyen, B. S. Gan, and S. Alexandrov. Post-buckling responses of elastoplastic FGM beams on nonlinear elastic foundation. Structural Engineering and Mechanics, 58, (3), (2016), pp. 515-532. https://doi.org/10.12989/sem.2016.58.3.515.

[19] D. K. Nguyen, K. V. Nguyen, B. S. Gan, and S. Alexandrov. Nonlinear bending of elastoplastic functionally graded ceramic-metal beams subjected to nonuniform distributed loads. Applied Mathematics and Computation, 333, (2018), pp. 443-459. https://doi.org/10.1016/j.amc.2018.03.100.

[20] P. K. Masjedi, A. Maheri, and P. M. Weaver. Large deflection of functionally graded porous beams based on a geometrically exact theory with a fully intrinsic formulation. Applied Mathematical Modelling, 76, (2019), pp. 938-957. https://doi.org/10.1016/j.apm.2019.07.018. 
[21] Y. Watanabe, Y. Inaguma, H. Sato, and E. Miura-Fujiwara. A novel fabrication method for functionally graded materials under centrifugal force: The centrifugal mixed-powder method. Materials, 2, (4), (2009), pp. 2510-2525. https://doi.org/10.3390/ma2042510.

[22] T. P. Vo, H. T. Thai, T. K. Nguyen, A. Maheri, and J. Lee. Finite element model for vibration and buckling of functionally graded sandwich beams based on a refined shear deformation theory. Engineering Structures, 64, (2014), pp. 12-22. https://doi.org/10.1016/j.engstruct.2014.01.029.

[23] T. P. Vo, H. T. Thai, T. K. Nguyen, F. Inam, and J. Lee. A quasi-3D theory for vibration and buckling of functionally graded sandwich beams. Composite Structures, 119, (2015), pp. 1-12. https://doi.org/10.1016/j.compstruct.2014.08.006.

[24] D. K. Nguyen and T. T. Tran. A corotational formulation for large displacement analysis of functionally graded sandwich beam and frame structures. Mathematical Problems in Engineering, 2016, (2016). https://doi.org/10.1155/2016/5698351.

[25] Y. S. Touloukian. Thermophysical properties of high temperature solid materials. Macmillan, New York, USA, (1967).

[26] S. S. Antman. Nonlinear problems of elasticity. Springer-Verlag, New York, (1995).

[27] C. Pacoste and A. Eriksson. Beam elements in instability problems. Computer Methods in Applied Mechanics and Engineering, 144, (1-2), (1997), pp. 163-197. https://doi.org/10.1016/s00457825(96)01165-6.

[28] E. N. Lages, G. H. Paulino, I. F. M. Menezes, and R. R. Silva. Nonlinear finite element analysis using an object-oriented philosophy-application to beam elements and to the cosserat continuum. Engineering with Computers, 15, (1), (1999), pp. 73-89. https://doi.org/10.1007/s003660050006.

[29] A. Mahi, E. A. A. Bedia, A. Tounsi, and I. Mechab. An analytical method for temperature-dependent free vibration analysis of functionally graded beams with general boundary conditions. Composite Structures, 92, (8), (2010), pp. 1877-1887. https://doi.org/10.1016/j.compstruct.2010.01.010.

[30] R. D. Cook, D. S. Malkus, and M. E. Plesha. Concepts and applications of finite element analysis. John Wiley \& Sons, New York, USA, 3rd edition, (1989).

[31] M. A. Crisfield. Non-linear finite element analysis of solids and structures, Vol. 1: Essentials. John Wiley \& Sons, Chichester, (1991).

[32] H. S. Shen and Z. X. Wang. Nonlinear analysis of shear deformable FGM beams resting on elastic foundations in thermal environments. International Journal of Mechanical Sciences, 81, (2014), pp. 195-206. https://doi.org/10.1016/j.ijmecsci.2014.02.020. 


\section{APPENDIX}

This Appendix presents detail expressions for the nodal forces and the tangent stiffness matrices in Eq. (19). The following notations are used

$$
\begin{aligned}
& s=\sin \bar{\theta}, \quad c=\cos \bar{\theta}, \\
& a_{1}=(s \bar{\varepsilon}-c \bar{\gamma}), \quad a_{2}=(c \bar{\varepsilon}+s \bar{\gamma}), \quad a_{3}=\bar{\gamma}^{2}-\bar{\varepsilon}(1+\bar{\varepsilon}), \\
& a_{4}=c \bar{\gamma}-s(1+\bar{\varepsilon}), \quad a_{5}=s \bar{\gamma}+c(1+\bar{\varepsilon}), \quad a_{6}=(1+\bar{\varepsilon})^{2}-\bar{\gamma}^{2} .
\end{aligned}
$$

The internal force vector

$$
\begin{aligned}
& \mathbf{f}_{a}=A_{11} \bar{\varepsilon}\left\{-c-s \frac{l}{2} \bar{\gamma} c s \frac{l}{2} \bar{\gamma}\right\}^{T}, \mathbf{f}_{b}=A_{22} \bar{\kappa}\left\{\begin{array}{llllll}
0 & 0 & 1 & 0 & 0 & -1
\end{array}\right\}^{T}, \\
& \mathbf{f}_{c}=A_{12} \bar{\varepsilon}\left\{\begin{array}{lllllll}
0 & 0 & 1 & 0 & 0 & -1
\end{array}\right\}^{T}+A_{12} \bar{\kappa}\left\{-c-s \frac{l}{2} \bar{\gamma} c c s \frac{l}{2} \bar{\gamma}\right\}^{T}, \\
& \mathbf{f}_{s}=\psi A_{33} \bar{\gamma}\left\{s-c-\frac{l}{2}(1+\bar{\varepsilon})-s c-\frac{l}{2}(1+\bar{\varepsilon})\right\}^{T}, \\
& \mathbf{f}_{T}=\left\{\begin{array}{llllll}
0 & -\frac{\left(w_{2}-w_{1}\right)}{l} N_{T} & 0 & 0 & \frac{\left(w_{2}-w_{1}\right)}{l} N_{T} & 0
\end{array}\right\}^{T},
\end{aligned}
$$

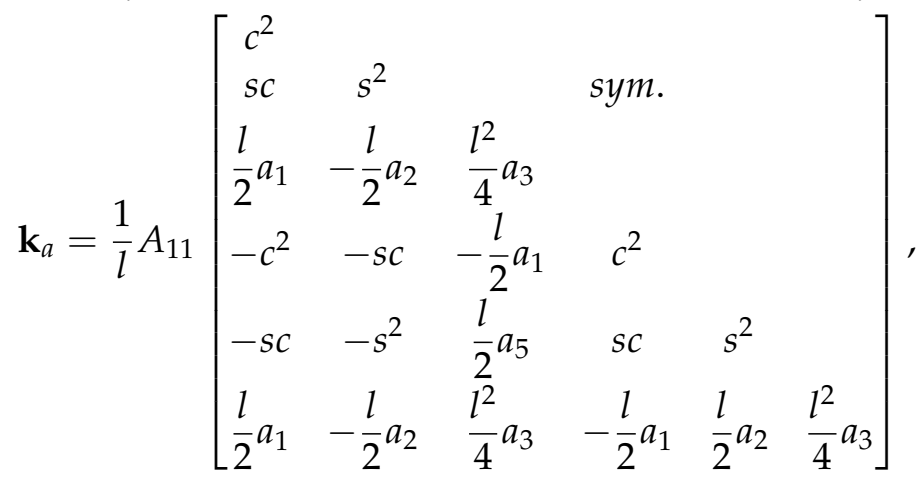

$$
\begin{aligned}
& \mathbf{k}_{b}=\frac{1}{l} A_{22}\left[\begin{array}{ccccccc}
0 & & & & & \\
0 & 0 & & \text { sym. } & & \\
0 & 0 & 1 & & & \\
0 & 0 & 0 & 0 & & \\
0 & 0 & 0 & 0 & 0 & \\
0 & 0 & -1 & 0 & 0 & 1
\end{array}\right] \text {, }
\end{aligned}
$$


$\mathbf{k}_{c}=A_{12} \bar{\kappa}\left[\begin{array}{cccccc}0 & 0 & s & 0 & 0 & s \\ 0 & 0 & -c & 0 & 0 & -c \\ s & -c & -\frac{l}{2}(1+\bar{\varepsilon}) & -\frac{s}{2} & \frac{c}{2} & \frac{l}{2}(1+\bar{\varepsilon}) \\ \frac{2}{2} & 0 & -s & 0 & 0 & -s \\ 0 & 0 & c & 0 & 0 & c \\ \frac{1}{2} s & -\frac{1}{2} c & -\frac{l}{2}(1+\bar{\varepsilon}) & -\frac{1}{2} s & \frac{1}{2} c & \frac{l}{2}(1+\bar{\varepsilon})\end{array}\right]+\frac{2}{l} A_{12}\left[\begin{array}{cccccc}0 & 0 & -c & 0 & 0 & c \\ 0 & 0 & -s & 0 & 0 & s \\ 0 & 0 & \frac{l}{2} \bar{\gamma} & 0 & 0 & -\frac{l}{2} \bar{\gamma} \\ 0 & 0 & c & 0 & 0 & -c \\ 0 & 0 & s & 0 & 0 & -s \\ 0 & 0 & \frac{l}{2} \bar{\gamma} & 0 & 0 & -\frac{l}{2} \bar{\gamma}\end{array}\right]$

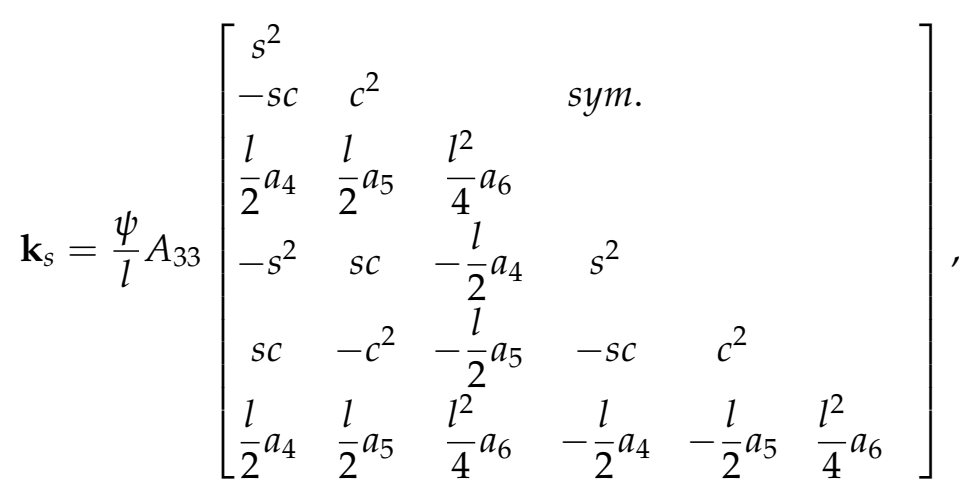

$\mathbf{k}_{T}=\frac{N_{T}}{l}\left[\begin{array}{cccccc}0 & & & & & \\ 0 & 1 & & \text { sym. } & & \\ 0 & 0 & 0 & & & \\ 0 & 0 & 0 & 0 & & \\ 0 & -1 & 0 & 0 & 1 & \\ 0 & 0 & 0 & 0 & 0 & 0\end{array}\right]$. 
